\title{
Observables in Topological Theories: A Superspace Formulation
}

\author{
José Luís Boldo ${ }^{\mathrm{a} *}$, Clisthenis P. Constantinidis ${ }^{\mathrm{a} *}$, François Gieres $^{\mathrm{b}}$, Matthieu Lefrançois ${ }^{\mathrm{b}}$ \\ and Olivier Piguet ${ }^{\mathrm{a} *}$ \\ ${ }^{a}$ Departamento de Física, CCE, Universidade Federal do Espírito Santo (UFES), \\ Av. Fernando Ferrari, s/n, BR-29060-900 - Vitória - ES (Brasil) \\ bInstitut de Physique Nucléaire, Université Claude Bernard (Lyon 1), \\ 43, boulevard du 11 novembre 1918, F - 69622 - Villeurbanne (France)
}

Observables of topological Yang-Mills theory were defined by Witten as the classes of an equivariant cohomology. We propose to define them alternatively as the BRST cohomology classes of a superspace version of the theory, where BRST invariance is associated to super Yang-Mills invariance. We provide and discuss the general solution of this cohomology. ${ }^{1}$

\section{Introduction}

Observables in topological theories are global, such as knot invariants in Chern-Simons theory, Wilson loops, etc. The problem of finding them all is a problem of "equivariant cohomology", as already pointed out by Witten in 1988 in the framework of 4-dimensional topological YangMills theory 123. Soon after, a superspace description of the latter model and its symmetries has been introduced 456.

Topological Yang-Mills theory is characterized by a local symmetry generator $\tilde{Q}$ which is nilpotent in a functional space constrained by gauge invariance, and equivariant cohomology is the cohomology of $\tilde{Q}$ restricted to this space. On the other hand, in the superspace approach which we shall use hereafter, the problem is transformed to that of looking for Yang-Mills BRST cohomology in a supersymmetric context where $\tilde{Q}$ corresponds to a strictly nilpotent supersymmetry generator $Q$.

Whereas the complete solution of equivariant cohomology is rather difficult to obtain [13], we

\footnotetext{
* Supported in part by the Conselho Nacional de Desenvolvimento Científico e Tecnológico (CNPq - Brazil).

${ }^{1}$ Prepared for International Conference on Renormalization Group and Anomalies in Gravity and Cosmology (IRGA 2003), Ouro Preto, MG, Brazil, 17-23 Mar 2003. Published in Nucl.Phys.Proc.Suppl.127 (2004) 30.
}

hope that our proposal of working with a superYang-Mills cohomology should simplify the task, and also make it more systematic and manageable for possible applications to more general models, such as topological gravity and models with more than one supersymmetry generator 7 .

An extended paper on this subject is available 8.

\section{Topological Yang-Mills Theory in Four Dimensions}

The purpose of four-dimensional topological Yang-Mills theory is to describe instanton or antiinstanton gauge field configurations. Denoting by $a=a_{\mu}^{a}(x) T_{a} d x^{\mu}$ the Yang-Mills gauge connection, where the matrices $T_{a}$ form a basis of the gauge Lie algebra, with

$\left[T_{a}, T_{b}\right]=f_{a b}{ }^{c} T_{c}, \quad \operatorname{Tr}\left(T_{a} T_{b}\right)=\delta_{a b}$,

we may write the instanton equation as the selfduality condition

$f_{\mu \nu}(x)=\frac{1}{2} \varepsilon_{\mu \nu \rho \sigma} f^{\rho \sigma}(x)$,

where $f_{\mu \nu}$ is the Yang-Mills field strength associated to the connection $a$, defined by

$f(a)=d a+a^{2}=\frac{1}{2} f_{\mu \nu}(x) d x^{\mu} d x^{\nu}$. 
Note that the self-duality condition (2) is obviously invariant under the usual (infinitesimal) Yang-Mills (YM) gauge transformation

$\delta_{(\omega)} a=d \omega+[a, \omega]$.

Witten's way [1] of generating these instanton configurations is to consider equation (2) as a gauge fixing condition for a local shift symmetry defined infinitesimally by its infinitesimal generator $\tilde{Q}$ :

$\tilde{Q} a(x)=\psi(x)$.

The coefficients $\psi_{\mu}(x)$ of the infinitesimal parameter 1-form $\psi$ are taken as anticommuting fields, i.e. as ghost fields, the generator $\tilde{Q}$ being thus interpreted as the BRST operator associated with the local shift symmetry. However, the transformation (5) is gauging away all 4 degrees of freedom of $a$, whereas there are only 3 independent equations in the condition (2). Therefore one has to gauge away one of the four degrees of freedom of $\psi$. This is done by imposing invariance under a local transformation of the ghost $\psi$ :

$\tilde{Q} \psi=-D_{a} \phi \equiv-d \phi-[a, \phi], \quad \tilde{Q} \phi=0$,

where the ghost for ghost $\phi(x)$ is a commuting 0 form. The $\tilde{Q}$-invariance of $\phi$ is needed for the sake of the nilpotency of the BRST operator $\tilde{Q}$, which however holds only up to a field dependent YMgauge transformation generalizing (4), defined by

$$
\begin{aligned}
& \delta_{(\omega)} a=d \omega+[a, \omega], \quad \delta_{(\omega)} \psi=[\psi, \omega], \\
& \delta_{(\omega)} \phi=[\phi, \omega],
\end{aligned}
$$

but with the infinitesimal parameter $\omega$ replaced by the ghost for ghost field $\phi$. One checks indeed that

$\tilde{Q}^{2}=\delta_{(\phi)}$.

However $\tilde{Q}$ is nilpotent when applied to YMgauge invariant quantities. This led Witten to define the observables of the theory as the cohomology classes of $\tilde{Q}$ in the space of the gauge invariant operators, which is a problem of equivariant cohomology [23]: an operator $\mathcal{O}$ belongs to the equivariant cohomology of $\tilde{Q}$ iff

$\tilde{Q} \mathcal{O}=0, \quad$ but $\mathcal{O} \neq \tilde{Q} \mathcal{P}$, with the conditions:

$\delta_{(\omega)} \mathcal{O}=0, \quad \delta_{(\omega)} \mathcal{P}=0$

To write an invariant action needs the introduction of other fields than $a, \psi, \phi$ which play the roles of antighosts and Lagrange multipliers for the self-duality constraint (2). This aspect of the theory will not be touched in the present contribution, the reader may consult the original literature [12]. We shall concentrate on the "geometrical sector" spanned by the connection $a$ and the ghosts $\psi$ and $\phi-$ which is what is needed for the determination of the observables.

\section{Superspace and Superforms}

The shift symmetry BRST operator $\tilde{Q}$ is odd, i.e. it transforms fermions into bosons and viceversa, hence it has the character of a supersymmetry (SUSY) generator. This has suggested, already a long time ago 456], a superspace and superfield description. Beyond a natural supersymmetry operation, whose generator is denoted by $Q$, the superspace formalism involves a superconnection and a supergauge invariance. The supergauge invariance may be partially fixed $\grave{a} l a$ Wess-Zumino by setting to zero certain components of the superconnection, leaving us with the field content described in the last section. The shift symmetry operator $\tilde{Q}$ defined by (56) is then recovered as the composition of the supersymmetry operation $Q$ with a particular field dependent supergauge transformation 4568.

Superspace is introduced by enlarging the $d$ dimensional spacetime, of coordinates $x^{\mu}(\mu=$ $0, \cdots, d-1)$, with the addition of one fermionic dimension $^{2}$ described by a Grassmann (i.e. anticommuting) coordinate $\theta$. One then defines a superfield as a superspace function

$S(x, \theta)=s(x)+\theta s^{\prime}(x)$,

linear in $\theta$ since $\theta^{2}=0$, which by definition transforms under an infinitesimal SUSY transformation as

$Q S=\partial_{\theta} S$,

2This construction may be generalized to the case of $N$ SUSY generators, in a superspace with $N$ fermionic dimensions [6]. 
or, in components: $Q s=s^{\prime}, \quad Q s^{\prime}=0$.

By construction the SUSY operator is strictly nilpotent: $Q^{2}=0$. Introducing the differential $d \theta$ - a commuting quantity - we may define $p$ superforms,

$\hat{\Omega}_{p}(x, \theta)=\sum_{k=0}^{p} \Omega_{p-k}(x, \theta)(d \theta)^{k}$,

where $\Omega_{p-k}(x, \theta)=\omega_{p-k}(x)+\theta \omega_{p-k}^{\prime}(x)$ is a $(p-k)$-form in $x$-space with superfield coefficients.

The superspace exterior derivative reads

$\hat{d}=d+d \theta \partial_{\theta}=d x^{\mu} \partial_{\mu}+d \theta \partial_{\theta}$, with $\hat{d}^{2}=0$.

A supergauge theory will be based on a superconnection, the 1-superform ${ }^{3}$

$$
\begin{aligned}
\hat{A} & =A(x, \theta)+d \theta A_{\theta}(x, \theta) \\
& =a(x)+\theta \psi(x)+d \theta(\chi(x)+\theta \phi(x)),
\end{aligned}
$$

a Faddeev-Popov ghost 0-superform

$C(x, \theta)=c(x)+\theta c^{\prime}(x)$,

and the corresponding BRST transformations

$\mathcal{S} \hat{A}=-(\hat{d} C+[\hat{A}, C]), \mathcal{S} C=-C^{2}\left(\mathcal{S}^{2}=0\right)$.

The latter read, in components:

$$
\begin{array}{ll}
\mathcal{S} a=-D c, & \mathcal{S} \psi=-[c, \psi]-D c^{\prime}, \\
\mathcal{S} \chi=-[c, \chi]-c^{\prime}, & \mathcal{S} \phi=-[c, \phi]-\left[\chi, c^{\prime}\right], \\
\mathcal{S} c=-c^{2}, & \mathcal{S} c^{\prime}=-\left[c, c^{\prime}\right] .
\end{array}
$$

The Wess-Zumino gauge mentioned at the beginning of this section consists in putting $\chi=0$. The $\tilde{Q}$-transformations of Section 2 are then reproduced by adding to the $Q$-transformations (9) a particular supergauge transformation, namely a BRST transformation (13) with $c=0$ and $c^{\prime}=\phi$. This shows the equivalence of the superspace approach with the formulation originally proposed by Witten, the latter being a Wess-Zumino gauge fixed version of the former. We shall however keep to the superspace formalism for the rest of this paper, only specializing to the Wess-Zumino gauge for comparisons with the literature.

${ }^{3}$ All forms and superforms in this paper are Lie algebra valued: $\Phi=\Phi^{a} T_{a}$, see [1].

\section{Observables in the Superspace Formal- ism}

By contrast to the equivariant cohomology defined by $\tilde{Q}$, the (unrestricted) cohomology of $Q$ is trivial: every form or superform which is $Q$ invariant is the $Q$-variation of another form or superform. This follows 910 from the fact that all fields are grouped in doublets $\left\{s(x), s^{\prime}(x)\right\}$ as in (10). However, the cohomology that is a priori not trivial is indeed the cohomology of the BRST operator (1213). This observation suggests to define an observable $\mathcal{O}_{(d)}$ as a BRST cohomology class:

$\mathcal{S} \mathcal{O}_{(d)}=0$, but $\mathcal{O}_{(d)} \neq \mathcal{S P}_{(d)}$,

where $\mathcal{O}_{(d)}$ and $\mathcal{P}_{(d)}$ are both $d$-dimensional ${ }^{4}$ space-time integrals:

$\mathcal{O}_{(d)}=\int_{M_{d}} \omega_{d}(x), \quad \mathcal{P}_{(d)}=\int_{M_{d}} \phi_{d}(x)$,

with the SUSY conditions:

$Q \mathcal{O}_{(d)}=0, \quad Q \mathcal{P}_{(d)}=0$.

The latter condition and the triviality of the cohomology of $Q$ implies now that, up to a possible total derivative which can be discarded without loss of generality, the integrand of $\mathcal{O}_{(d)}-$ the $d$ form $\omega_{d}$ - is $Q$-exact:

$\omega_{d}(x)=Q \Omega_{d}(x, \theta)$,

hence

$\mathcal{O}_{(d)}=\int_{M_{d}} Q \Omega_{d}(x, \theta)=\int_{M_{d}} \partial_{\theta} \Omega_{d}(x, \theta)$,

which is the superspace integral of a superfield form, i.e. of a space-time form whose coefficients are superfields. From now on we shall work with such superfield forms.

\section{General Solution}

We want to find the general solution of the BRST cohomology problem (14) with the SUSY invariance condition (15). The latter condition

\footnotetext{
$\overline{4}$ The space-time dimension will not be fixed a priori. The space-time integrals are performed over an arbitrary $d$ manifold $M_{d}$.
} 
being automatically fulfilled using superspace integrals such as (16), we are left with the BRST cohomology equation (14). It implies for the integrand $\Omega_{d} \equiv{ }^{D-d} \Omega_{d}^{0}$ in (16) the condition

$\mathcal{S}^{D-d} \Omega_{d}^{0}+d^{D-d} \Omega_{d-1}^{1}+Q^{D-d-1} \Omega_{d}^{1}=0$,

where ${ }^{D-d} \Omega_{d-1}^{1}$ and ${ }^{D-d-1} \Omega_{d}^{1}$ are some superfield forms. The indices $p, g$ and $s$ of ${ }^{s} \Omega_{p}^{g}$ mean, respectively, its space-time form degree, ghost number and SUSY number. The ghost number is equal to the degree in the superfield ghost $C$ or its components $c, c^{\prime}$. The SUSY number, which is indeed the "ghost number" corresponding to local shift symmetry, is defined by giving the value $s=1$ to the SUSY operator $Q$ (hence $s=-1$ for the $\theta$ coordinate) and $s=0$ for the Yang-Mills connection $a(x)$. All the forms $\Omega$. are taken as polynomials in the superfields $A, A_{\theta}, C$ and their derivatives $d(\cdots), \partial_{\theta}(\cdots)$, which defines a functional space denoted by $\mathcal{E}_{\mathrm{S}}$.

Now, nilpotency of $\mathcal{S}$ together with the cohomological triviality of $d$ and $Q$ in the space $\mathcal{E}_{\mathrm{S}}$ imply that (17) is but the first one of a set of bi-descent equations [8]:

$$
\begin{aligned}
& \mathcal{S}^{D-p-g} \Omega_{p}^{g}+d^{D-p-g} \Omega_{p-1}^{g+1} \\
& +Q^{D-p-g-1} \Omega_{p}^{g+1}=0 \\
& (p=0, \ldots, d ; g=d-p, \ldots, D-p) .
\end{aligned}
$$

The observables of dimension $d$ and SUSY number $s$ will thus be given by (16) as the general solution ${ }^{s-1} \Omega_{d}^{0}$ of (18), at ghost number 0, i.e. with $D=d+s-1$.

The general solution of the bi-descent equations (18) is divided in the two classes described in the two following subsections [8].

\subsection{Equivariantly trivial solutions}

The first class of nontrivial solutions of the bi-descent equations correspond to nontrivial solutions of the BRST cohomology problem (14) which are trivial in the sense of the equivariant cohomology. They have the general form [ 8

$$
\begin{aligned}
& { }^{D-d+1} \mathcal{O}_{(d)} \\
= & \int_{M_{d}} Q^{D-d} \mathcal{H}_{d}\left(F_{A}, \Psi, \Phi, D_{A} \Psi, D_{A} \Phi\right),
\end{aligned}
$$

where $\mathcal{H}_{d}$ is a gauge invariant function of the superfield forms $F_{A}, \Psi$ and $\Phi$ and their covariant derivatives $D_{A} \Psi=d \Psi+[A, \Psi]$ and $D_{A} \Phi$. These superfield forms are the components of the supercurvature $\hat{F} \equiv \hat{d} \hat{A}+\hat{A}^{2}$,

$\hat{F}=F_{A}+\Psi d \theta+\Phi(d \theta)^{2}$,

with

$$
\begin{aligned}
& F_{A}=d A+A^{2}, \\
& \Psi=\partial_{\theta} A+D_{A} A_{\theta}=\psi+D_{a} \chi+O(\theta), \\
& \Phi=\partial_{\theta} A_{\theta}+A_{\theta}^{2}=\phi+\chi^{2}+O(\theta) .
\end{aligned}
$$

Their BRST transformations read as

$$
\begin{aligned}
& \mathcal{S} F_{A}=-\left[C, F_{A}\right], \quad \mathcal{S} \Psi=-[C, \Psi], \\
& \mathcal{S} \Phi=-[C, \Phi] .
\end{aligned}
$$

The solutions (19), although nontrivial in the sense of the BRST cohomology, are trivial in the sense of the equivariant cohomology. An easy way to see this is to go to the Wess-Zumino gauge, where they conserve the same form as in (19), but with $A, F_{A}, \Psi$ and $\Phi$ replaced by $a, F_{a}=d a+a^{2}$, $\psi$ and $\phi$, and the operator $Q$ replaced by $\tilde{Q}$. The result is then explicitly given by the $\tilde{Q}$-variation of a gauge invariant integral.

\subsection{Equivariantly nontrivial solutions}

The second class of nontrivial solutions of the bi-descent equations (18) corresponds to nontrivial solutions of the BRST cohomology problem (14) which are also nontrivial in the sense of the equivariant cohomology. They are given in terms of superforms by the following superspace algorithm 8 .

1. Consider all the superforms $\hat{\Omega}_{D}(x, \theta)$ (of ghost number 0 and superform degree $D$ ) which are nontrivial elements of the cohomology $H(\mathcal{S} \mid \hat{d})$ of $\mathcal{S}$ modulo $\hat{d}$ in the space of the superforms consisting of polynomials of the basic superforms $\hat{A}(x, \theta), C(x, \theta), \hat{d} \hat{A}(x, \theta)$ and $\hat{d} C(x, \theta)$. Nontriviality in the sense of the cohomology $H(\mathcal{S} \mid \hat{d})$ for a superform $\hat{\Omega}$ means

$\mathcal{S} \hat{\Omega}=0 \quad($ modulo $\hat{d})$,

but $\quad \hat{\Omega} \neq \mathcal{S} \hat{\Phi} \quad$ (modulo $\hat{d}$ ). 
These $\hat{\Omega}_{D}$ are nontrivial solutions of sets of "superdescent equations"

$$
\begin{aligned}
\mathcal{S} \hat{\Omega}_{D}+\hat{d} \hat{\Omega}_{D-1}^{1} & =0, \mathcal{S} \hat{\Omega}_{D-1}^{1}+\hat{d} \hat{\Omega}_{D-2}^{2}=0 \\
\cdots, \quad \mathcal{S} \hat{\Omega}_{0}^{D} & =0 .
\end{aligned}
$$

2. Expand $Q \hat{\Omega}_{D}=\partial_{\theta} \hat{\Omega}_{D}$ according to the space-time form degree $p$ :

$Q \hat{\Omega}_{D}=\sum_{p=0}^{D} w_{p}(x)(d \theta)^{D-p}$.

The space-time forms $w_{p}$ are our solutions. Indeed,

$\mathcal{S} w_{p}(x)=0$ (modulo $\left.d\right)$ and $Q w_{p}(x)=0$,

which follows from applying the operator $Q$ to the first of the superdescent equations (21), and using the identities $Q \hat{d}=-\hat{d} Q=-d Q$, which are direct consequences of the definition (11).

We thus obtain, for a given maximum degree $D$, a set of observables

$\mathcal{O}_{(p)}=\int_{M_{p}} w_{p}(x) \quad(p=0, \ldots D)$,

where the space-time forms $w_{p}$ are the coefficients of the superform $Q \hat{\Omega}_{D}$, with $\hat{\Omega}_{D}$ representing a nontrivial solution of the superdescent equations (21).

\subsection{Solution of the superdescent equation}

The nontrivial observables are thus completely determined from the general solution of the superdescent equations (21). The latter is given by the generalization to the present superspace formalism of standard results of BRST cohomology [10]. The result is:

$$
\begin{aligned}
& \hat{\Omega}_{D}=\theta_{r_{1}}^{\mathrm{CS}}(\hat{A}) f_{r_{2}}(\hat{F}) \cdots f_{r_{L}}(\hat{F}), \\
& \text { with } \quad D=2 \sum_{i=1}^{L} m_{r_{i}}-1, \quad L \geq 1,
\end{aligned}
$$

where $f_{r}(\hat{F})$ is the supercurvature invariant of degree $m_{r}$ in $\hat{F}$ corresponding to the gauge group Casimir operator of degree $m_{r}$, and $\theta_{r}^{\mathrm{CS}}(\hat{A})$ is the associated Chern-Simons form:

$\hat{d} \theta_{r}^{\mathrm{CS}}(\hat{A})=f_{r}(\hat{F})$.
We note that the superform degree of the solution (24) is odd.

An equivalent and convenient way to write down the integrants $w_{p}(x)$ of the observables is given by the following expansion of the super exterior derivative of the superform (24):

$$
\begin{aligned}
\hat{d} \hat{\Omega}_{D}= & f_{r_{1}}(\hat{F}) \cdots f_{r_{L}}(\hat{F}) \\
= & f_{r_{1}}\left(F_{A}\right) \cdots f_{r_{L}}\left(F_{A}\right) \\
& +\sum_{p=0}^{D}{ }^{D+1-p} W_{p}^{0}(d \theta)^{D+1-p},
\end{aligned}
$$

the first equality following from (25). It is indeed easy to check that the forms

$w_{p}^{\prime}(x)=\left.{ }^{D-p+1} W_{p}^{0}\right|_{\theta=0}$

differ from the $w_{p}(x)$ by derivative terms only, and thus may be substituted to the latter in the integrals (23) defining the observables.

One finally checks that, upon reducing the results to the Wess-Zumino gauge, one recovers the observables originally given by Witten [1].

\section{Example}

We consider the case of maximum degree $D=$ 3 . The superdescent equations read as

$$
\begin{array}{ll}
\mathcal{S} \hat{\Omega}_{3}+\hat{d} \hat{\Omega}_{2}^{1}=0, & \mathcal{S} \hat{\Omega}_{2}^{1}+\hat{d} \hat{\Omega}_{1}^{2}=0, \\
\mathcal{S} \hat{\Omega}_{1}^{2}+\hat{d} \hat{\Omega}_{0}^{3}=0, & \mathcal{S} \hat{\Omega}_{0}^{3}=0 .
\end{array}
$$

The unique nontrivial solution is

$$
\begin{array}{ll}
\hat{\Omega}_{3}=\operatorname{Tr}\left(\hat{A} \hat{d} \hat{A}+\frac{2}{3} \hat{A}^{3}\right), & \hat{\Omega}_{2}^{1}=\operatorname{Tr}(\hat{A} \hat{d} C), \\
\hat{\Omega}_{1}^{2}=\operatorname{Tr}(C \hat{d} C), & \hat{\Omega}_{0}^{3}=-\frac{1}{3} \operatorname{Tr} C^{3} .
\end{array}
$$

Note that $\hat{\Omega}_{3}$ is the Chern-Simons superform associated to the quadratic Casimir operator of the gauge group. The observables are then given by the expansion

$$
\begin{aligned}
\left.\hat{d} \hat{\Omega}_{3}^{0}\right|_{\theta=0} & =\left.\operatorname{Tr} \hat{F}^{2}\right|_{\theta=0} \\
& =\operatorname{Tr} F_{a}{ }^{2}+\sum_{p=0}^{3} w_{p}^{\prime}(d \theta)^{4-p},
\end{aligned}
$$

with

$$
\begin{aligned}
& w_{0}^{\prime}=\operatorname{Tr}\left(\phi^{2}+2 \phi \chi^{2}\right) \\
& w_{1}^{\prime}=2 \operatorname{Tr}\left(\psi \phi+\psi \chi^{2}+\phi D_{a} \chi\right)+d(\cdots), \\
& w_{2}^{\prime}=\operatorname{Tr}\left(\psi^{2}+2 \phi F_{a}+2 \psi D_{a} \chi\right)+d(\cdots) \\
& w_{3}^{\prime}=2 \operatorname{Tr}\left(\psi F_{a}\right)+d(\cdots) .
\end{aligned}
$$


The observables are the integrals of these forms (and of $\operatorname{Tr} F_{a}{ }^{2}$ ) on closed submanifolds of appropriate dimension.

In the Wess-Zumino gauge:

$$
\begin{aligned}
& w_{0}^{\prime}=\operatorname{Tr}\left(\phi^{2}\right) \\
& w_{1}^{\prime}=2 \operatorname{Tr}(\psi \phi)+d(\cdots) \\
& w_{2}^{\prime}=\operatorname{Tr}\left(2 \phi F_{a}+\psi^{2}\right)+d(\cdots), \\
& w_{3}^{\prime}=2 \operatorname{Tr}\left(\psi F_{a}\right)+d(\cdots)
\end{aligned}
$$

which corresponds to Witten's result up to total derivatives.

\section{Conclusion}

The superspace BRST cohomology which we have proposed as an alternative definition of the observables of a topological theory of the YangMills type, reproduces Witten's original result using a rather straightforward extension to superspace of standard results on BRST cohomology 10. We have also seen that this cohomology produces other solutions, which are easily proved to be trivial in the sense of equivariant cohomology. The main achievement has been to show [8] that there are no other solutions to the problem of the superspace BRST cohomology. These results lead us to wonder about the applicability of such an approach to the construction of observables in more complex systems, for example topological gravity and Yang-Mills theories with more than one supersymmetry generator. These are problems under current investigation, and we hope to provide answers to these questions soon [7].

\section{Acknowledgments}

J.L.B., C.P.C. and O.P. thank the Conselho Nacional de Desenvolvimento Científico e Tecnoló-gi-co ( $\mathrm{CNPq}$ - Brazil) for financial support. C.P.C. also thanks the Coordenação de Aperfeiçoamento de Pessoal de Nível Superior (CAPES - Brazil) for financial support, and the Abdus Salam International Center for Theoretical Physics (ICTP - Italy) for hospitality during a three months stay under its Associate Program. O.P. acknowledges the Institut de Physique Nucléaire, Université Claude Bernard (Lyon 1) for its kind hospitality during a one month's stay as Professeur Invité.

\section{REFERENCES}

1. E. Witten, Int.J.Mod.Phys. A6 (1991) 2775; Commun.Math.Phys. 117 (1988) 353.

2. P. van Baal, S. Ouvry and R. Stora, Phys. Lett. B220 (1989) 159.

3. L. Baulieu and I.M. Singer, Nucl. Phys. (Proc. Suppl.) B5 (1988) 12.

H. Kanno, Z.Physik C43 (1989) 477.

A. Blasi and R. Collina, Phys.Lett. B222 (1989) 419.

R. Stora, F. Thuillier and J. C. Wallet, "Algebraic structure of cohomological field theory models and equivariant cohomology", Lectures at the Caribbean Spring School of Mathematics and Theoretical Physics, St. François, Guadeloupe, May 30 - June 13, 1993, preprint ENSLAPP-A-481-94,

F. Delduc, N. Maggiore, O. Piguet and S. Wolf, Phys. Lett. B385 (1996) 132, [hep-th/9605158] .

4. J.H. Horne, Nucl. Phys. B318 (1989) 22.

5. C. Aragão de Carvalho and L. Baulieu, Phys. Lett. B275 (1992) 323.

6. M. Blau and G. Thompson, Commun. Math. Phys. 152 (1993) 41, [hep-th/9112012] ;

Nucl. Phys. B492 (1997) 545, [hep-th/9612143] .

7. J.L. Boldo, C.P. Constantinidis, F. Gieres, M. Lefrançois, J.A. Nogueira and O. Piguet, work in progress.

8. J.L. Boldo, C.P. Constantinidis, F. Gieres, M. Lefrançois and O. Piguet, "Observables in topological Yang-Mills Theories", [hep-th/0303053]

and "Topological Yang-Mills theories and their observables: a superspace approach", Proceedings of the 2nd International Londrina Winter School Mathematical Methods in Physics, 2002, Londrina, Brazil, to appear in Int. J. Mod. Phys. A, [hep-th/0303084] .

9. O. Piguet and S.P. Sorella, "Algebraic Renormalization", Lecture Notes in Physics m28, (Springer-Verlag, Berlin 1995).

10. G. Barnich, F. Brandt and M. Henneaux, Phys. Rep. 338 (2000) 439. 